\title{
A Comparative Study on the Treatment of Osteoporotic Vertebral Compression Fracture with Different Injection Volumes of High Viscosity Bone Cement in Percutaneous Vertebroplasty
}

\author{
S. SONG ${ }^{1}$, S. LIU1 1 , Y. K. WANG ${ }^{1}$ AND C. Q. ZHANG ${ }^{1 *}$
}

${ }^{1}$ Department of Orthopedics, The Third Affiliated Hospital of Soochow University, Suzhou, '2Department of Orthopedics, Shanghai Jiaotong University Affiliated Sixth People's Hospital, Shanghai, P. R. China

\section{Song et al.: Comparative Treatment of Osteoporotic Vertebral Compression Fracture}

\begin{abstract}
This study was to compare the clinical effect of different injection volumes of high viscosity bone cement in percutaneous vertebroplasty on the treatment of osteoporotic vertebral compression fractures. One hundred and fifty cases of patients with osteoporotic vertebral compression fractures treated in the Third Affiliated Hospital of Soochow University from January 2017 to October 2018 were enrolled and randomly divided into the high dose group (50 cases), the middle dose group (50 cases) and the low dose group (50 cases) according to the injection volumes of high viscosity bone cement in percutaneous vertebroplasty. There were no significant differences on single vertebral operation time and bleeding volume among the 3 groups. After percutaneous vertebroplasty treatment, the visual analogue score and Oswestry disability index were significantly improved 5 days and 12 months later in all 3 groups. However, no significant differences were found on the visual analogue score and Oswestry disability index among these 3 groups before surgery and 5 days and 12 months later. The vertebral height and Cobb angle of injured vertebrae were significantly improved 5 days and 3 months after operation in the all 3 groups. However, the improvement had no correlation with the dose of high viscosity bone cement. After treatment, there were 14, 9 and 2 cases of bone cement leakage in high dose, middle dose and low dose groups, respectively. The leak rates were 28, 18 and $4 \%$ and there were significant differences among the 3 groups. In conclusion, different injection volumes of high viscosity bone cement in percutaneous vertebroplasty all reached a good effect in the treatment of osteoporotic vertebral compression fractures. And low dose could significantly reduce the leakage of bone cement. Therefore, low dose of high viscosity bone cement could be the first choice for the treatment of osteoporotic vertebral compression fractures for its safety.
\end{abstract}

Key words: High viscosity bone cement, percutaneous vertebroplasty, osteoporotic vertebral compression fracture, Cobb angle

Osteoporotic vertebral compression fractures (OVCFs) are common fractures in the elderly. The most common symptom is severe pain in the back and lumbar spine, which severely restricted their actions. There are 1.4 million patients of vertebral compression fractures received clinical treatment all of the world per year and most patients can obtain satisfactory results by conservative or surgical treatment. However, conservative treatment requires long-term bed rest, which will aggravate osteoporosis, increase the risk of complications and death ${ }^{[1,2]}$. Percutaneous vertebroplasty (PVP) is an important minimally invasive surgical treatment for OVCFs and has the advantages of fast pain relief and early functional exercise. Nevertheless, the leakage rate of bone cement after PVP can reach 25.8-65\%. Nowadays, there is still no consensus on how to reduce bone cement leakage. A small amount of bone cement leakage generally does not cause clinical symptoms. But if bone cement seeps into nerve root or big blood vessel, it can cause heat injury and compression of nerve root and blood vessel, pulmonary embolism and other serious complications. Though the efficacy of low viscosity and high viscosity bone cement for the treatment of OVCFs is similar in $\mathrm{PVP}^{[3]}$, the leakage rate of low viscosity bone cement in vein and paravertebral was higher than that in viscosity bone cement ${ }^{[4,5]}$. However, the current clinical application of high viscosity bone cement in the treatment of vertebral 
compression fractures in China is still not mature enough and the injection volume of high viscosity bone cement is controversial. The purpose of this study was to compare the clinical effect of different injection volumes of high viscosity bone cement in PVP on the treatment of OVCFs, so as to find an effective treatment scheme to reduce the postoperative leakage rate.

One hundred and fifty cases of patients with OVCFs treated in the Third Affiliated Hospital of Soochow University from January 2017 to October 2018 were enrolled, including 78 males and 72 females, aged at $60-79 \mathrm{y}$ with a mean age at $71.3 \pm 2.5 \mathrm{y}$. Vertebral collapse degree was $52.8 \pm 7.6 \%$. Inclusion criteria were bone mineral density examination indicated osteoporosis; all patients underwent strict physical examination and imaging examination to confirm a diagnosis of thoracolumbar vertebral compression fractures. Persistent lumbar and back pain and limited activity despite of many treatments such as drugs and physical therapy and no spinal cord or nerve root injury or compression symptoms and signs; CT showed no obvious damage to the posterior wall of the vertebral body compression, vertebral collapse degree was less than $75 \%$; without coagulation dysfunction contraindication. Exclusion criteria were patients with spinal cord injury, cauda equina injury or neurological injury; accompanied by spinal stenosis, spinal tuberculosis and spinal cord tumor; vertebral fracture line across the vertebral or incomplete vertebral; accompanied by severe hypertension, diabetes, coronary heart disease and other major diseases, cannot afford surgery according to systemic evaluation. The 150 patients were randomly divided into the high dose group (50 cases), the medium dose group (50 cases) and low the dose group (50 cases). There were no significant differences in gender, age and disease condition between all the 3 groups. All patients or their families have signed informed consent.

The patients were taken prone position with electrocardiogram monitoring, local anesthesia and fluoroscopy positioning using $\mathrm{C}$ arm. Inserting needle from the more severe compression side and performing surgery using bilateral percutaneous pedicle approach. The injection cannula was attached to the high viscosity bone cement and injected with a pressure injection pump. The whole injection was performed under the fluoroscopy, and the injection was stopped immediately after the leakage from the posterior edge of the vertebral body occurring. Injection volume of vertebral bone cement in the high dose group was 5.0-7.0 ml; injection volume of vertebral bone cement in the median dose group was 3.0-5.0 ml; Injection volume of vertebral bone cement in the low dose group was less than $3.0 \mathrm{ml}$. Bone cement is high viscosity bone cement from Palacos $R+G$ in German.

The visual analogue score (VAS) and Oswestry disability index (ODI) were used to evaluate the pain degree and motor function before, $5 \mathrm{~d}$ and 12 mo after surgery. Measuring vertebral height and the Cobb's angel with CT measurements before, $5 \mathrm{~d}$ and 3 mo after surgery. Record and analyze the occurrence of bone cement leakage and other complications. SPSS13.0 statistical software was adopted for statistical analysis. The measurement data were expressed as mean \pm standard deviation. Comparisons among the three groups were conducted using one-way ANOVA. Enumeration data were expressed by percentage (\%) and comparisons on enumeration data were conducted using Chi-Square test. $\mathrm{p}<0.05$ means a significant difference. All the 150 patients received the operation successfully. Injection volume of bone cement was $5.3 \pm 0.5 \mathrm{ml}$ in high dose group, $4.3 \pm 0.2 \mathrm{ml}$ in middle dose group and $2.3 \pm 0.4 \mathrm{ml}$ in low dose group. There were significant differences on the injection volume of bone cement among the three groups. There were no significant differences on operation time and bleeding volume, as shown in Table 1. Typical cases in each group were shown in fig 1.

After PVP treatment, the VAS and ODI were significantly improved $5 \mathrm{~d}$ and 12 mo later in all 3 groups. However, no significant differences were found on the VAS score

TABLE 1: COMPARISONS ON GENERAL CONDITION AMONG THE THREE GROUPS

\begin{tabular}{lcccc}
\hline Group & N & $\begin{array}{c}\text { Operation } \\
\text { time }(\mathrm{min})\end{array}$ & $\begin{array}{c}\text { Bleeding } \\
(\mathrm{ml})\end{array}$ & $\begin{array}{c}\text { Injection } \\
\text { volumes }(\mathrm{ml})\end{array}$ \\
\hline High dose & 50 & $47.3 \pm 3.6$ & $9.2 \pm 1.5$ & $5.3 \pm 0.5$ \\
Median dose & 50 & $46.9 \pm 4.5$ & $9.3 \pm 1.2$ & $4.3 \pm 0.2$ \\
Low dose & 50 & $47.1 \pm 3.4$ & $9.2 \pm 1.4$ & $2.3 \pm 0.4$ \\
F & & 1.267 & 0.975 & 1.032 \\
$\mathrm{P}$ & & $>0.05$ & $>0.05$ & $>0.05$ \\
\hline
\end{tabular}

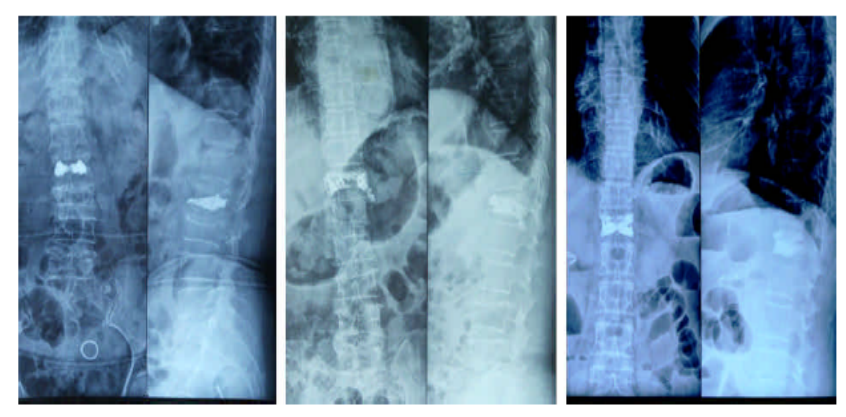

Fig. 1: The positive and lateral radiographs after percutaneous vertebroplasty 
and ODI among these 3 groups before surgery and $5 \mathrm{~d}$ and $12 \mathrm{~m}$ later, as shown in Table 2 . The vertebral height and Cobb angle of injured vertebrae were significantly improved $5 \mathrm{~d}$ and 3 mo after operation in all 3 groups. However, the improvement had no correlation with the dose of high viscosity bone cement, as shown in Table 3. After treatment, there were 14, 9 and 2 cases of bone cement leakage in high dose, middle dose and low dose groups, respectively. The leak rates were 28,18 and $4 \%$ and there were significant differences among the 3 groups. Low dose group has the least leakage rate, while high dose group has the highest leakage rate, as shown in Table 4.

Compared with the traditional treatment, PVP has been widely used because of its advantages of less trauma, quick response and less complications ${ }^{[6]}$. Bone cement leakage is one of the most common and most serious complications in PVP treatment and may cause catastrophic damage to the spinal cord and nerve root, and may even lead to life-threatening pulmonary embolism. With the maturity and wide application of this technology, the extravasation of bone cement is mainly prevented by the choice of bone cement material, the improvement of surgical instruments, the dosage and time of bone cement injection ${ }^{[7]}$. High viscosity bone cement possesses instant high viscosity, low polymerization temperature and can be injected longtime ${ }^{[8]}$. It can reduce the polymerization effects of bone cement and thermal of bone cement leakage in the PVP treatment, which may greatly improve the safety of PVP, so as to avoid the occurrence of nerve injury and pulmonary embolism. In fact, high viscosity bone cement has higher safety and efficacy than traditional bone cement in the treatment of $\mathrm{OVCF}^{[9]}$. The most common and serious complication of bone cement in the treatment of vertebral compression fractures is the loosening of the prosthesis. The medium and long-term prosthesis loosening rate in the use of high viscosity bone cement is much lower than the use of ordinary bone cement, and appropriate rehabilitation treatment is helpful to reduce the incidence of medium and longterm complications ${ }^{[10,11]}$.

It is still controversial on the injection volume on high viscosity bone cement for the treatment, clinical study showed that there was no significant correlation between the injection volume of bone cement and clinical efficacy. A vertebral body filling rate less than $30 \%$ can reduce the pain and stability of vertebral fracture ${ }^{[12-15]}$. In our study, there were 14,9 and 2 cases of bone cement leakage in high dose, middle dose and low dose group respectively. The leak rates were 28, 18 and $4 \%$ and there were significant differences among the 3 groups. The lowest leak rate was only $4 \%$ when the injection volume does not exceed the $3.0 \mathrm{ml}$, which showed a reduction in bone cement leakage rate when reducing the amount of bone cement injection.

TABLE 2: COMPARISONS ON VAS SCORE AND ODI AMONG THE THREE GROUPS

\begin{tabular}{|c|c|c|c|c|c|c|c|c|c|}
\hline \multirow{2}{*}{ Group } & \multirow{2}{*}{$\mathbf{N}$} & \multicolumn{3}{|c|}{ VAS } & \multirow{2}{*}{$\mathbf{P}$} & \multicolumn{3}{|c|}{ ODI (\%) } & \multirow{2}{*}{$\mathbf{P}$} \\
\hline & & Before & $5 \mathrm{~d}$ & $12 \mathrm{mo}$ & & Before & $5 d$ & $12 \mathrm{mo}$ & \\
\hline High dose & 50 & $8.1 \pm 1.3$ & $2.9 \pm 1.2$ & $2.1 \pm 0.6$ & $<0.05$ & $67.8 \pm 9.7$ & $46.1 \pm 6.8$ & $30.4 \pm 4.5$ & $<0.05$ \\
\hline Median dose & 50 & $7.9 \pm 1.2$ & $2.9 \pm 0.9$ & $2.0 \pm 0.7$ & $<0.05$ & $65.5 \pm 8.7$ & $44.7 \pm 5.4$ & $29.6 \pm 5.3$ & $<0.05$ \\
\hline Low dose & 50 & $8.0 \pm 1.3$ & $2.9 \pm 1.0$ & $2.0 \pm 0.8$ & $<0.05$ & $66.9 \pm 9.2$ & $46.7 \pm 7.7$ & $29.4 \pm 6.1$ & $<0.05$ \\
\hline $\mathrm{P}$ & & $>0.05$ & $>0.05$ & $>0.05$ & & $>0.05$ & $>0.05$ & $>0.05$ & \\
\hline
\end{tabular}

TABLE 3: COMPARISONS ON VERTEBRAL HEIGHT AND THE COBB'S ANGEL AMONG GROUPS

\begin{tabular}{|c|c|c|c|c|c|c|c|c|c|}
\hline \multirow{2}{*}{ Group } & \multirow{2}{*}{$\mathbf{N}$} & \multicolumn{3}{|c|}{ Vertebral height (mm) } & \multirow{2}{*}{$\mathbf{P}$} & \multicolumn{3}{|c|}{ Cobb's angel } & \multirow{2}{*}{$\mathbf{P}$} \\
\hline & & Before & $5 d$ & $3 \mathrm{mo}$ & & Before & $5 \mathrm{~d}$ & $3 \mathrm{mo}$ & \\
\hline High dose & 50 & $8.0 \pm 1.2$ & $3.1 \pm 1.3$ & $2.2 \pm 0.3$ & $<0.05$ & $66.6 \pm 10.2$ & $45.4 \pm 6.2$ & $30.1 \pm 4.3$ & $<0.05$ \\
\hline Median dose & 50 & $7.8 \pm 1.4$ & $3.0 \pm 0.6$ & $2.0 \pm 0.4$ & $<0.05$ & $64.1 \pm 7.8$ & $43.5 \pm 5.1$ & $28.5 \pm 5.1$ & $<0.05$ \\
\hline Low dose & 50 & $8.1 \pm 1.1$ & $2.9 \pm 1.1$ & $2.0 \pm 0.5$ & $<0.05$ & $66.2 \pm 9.2$ & $46.8 \pm 4.9$ & $29.1 \pm 5.7$ & $<0.05$ \\
\hline$P$ & & $>0.05$ & $>0.05$ & $>0.05$ & & $>0.05$ & $>0.05$ & $>0.05$ & \\
\hline
\end{tabular}

TABLE 4: COMPARISONS ON BONE CEMENT LEAKAGE AMONG THE THREE GROUPS

\begin{tabular}{lccccc}
\hline Group & N & Intervertebral disc leakage* & Venous leakage* & Paravertebral leakage* & Leakage rate* $^{*}$ \\
\hline High dose & 50 & $5(10)$ & $4(8)$ & $5(10)$ & $14(28)$ \\
Median dose & 50 & $4(8)$ & $3(6)$ & $2(4)$ & $9(18)$ \\
Low dose & 50 & $1(2)$ & - & $1(2)$ & $2(4)$ \\
$X^{2}$ & & & & 10.464 \\
P & & & & $<0.01$ \\
\hline
\end{tabular}

Number of cases and the numerals in parenthesis is \% leakage 
VAS and ODI improved significantly in low dose bone cement injection group $5 \mathrm{~d}$ and 12 mo after the surgery when compared with preoperative evaluation. But there was no significant difference when compared with high and middle dose of bone cement injection group. Besides, the vertebral height and Cobb angle of injured vertebrae were significantly improved 5 $\mathrm{d}$ and 3 mo after operation in all 3 groups. However, the improvement had no correlation with the dose of high viscosity bone cement. These results confirmed that there was no significant correlation between the bone cement injection volume and clinical efficacy. It can also achieve good curative effect with an injection volume less than $3.0 \mathrm{ml}$.

In conclusion, different injection volumes of high viscosity bone cement in PVP all reached a good effect in the treatment of OVCFs and low dose could significantly reduce the leakage of bone cement. Therefore, low dose of high viscosity bone cement could be the first choice for the treatment of OVCFs for its safety.

\section{Conflict of interest:}

No conflict of interest between any of the authors.

\section{REFERENCES}

1. Lador R, Liberman S, Ben-Galim P, Dreiangel N, Reitman CA, Hipp JA. A cadaver study to compare vertebral augmentation with high-viscosity cement to augmentation with conventional lower -viscosity cement. J Spinal Disord Tech 2013;26:68-73.

2. Georgy BA. Clinical experience with high-viscosity cements for percutaneous vertebral body augmentation: occurrence, degree, and location of cement leakage compared with kyphoplasty. AJNR Am J Neuroradiol 2010;31:504-8.

3. Wang H, Sribastav SS, Ye F, Yang C, Wang J, Liu H, et al. Comparison of highviscosity cement vertebroplasty and balloon kyphoplasty for the treatment of osteoporotic vertebral compression fractures. Pain Physician 2015;18:E187-94.

4. Trumm CG, Jakobs TF, Stahl R, Sandner TA, Paprottka PM, Reiser MF, et al. CT fluoroscopy-guided vertebral augmentation with a radiofrequency -induced, high-viscosity bone cement (StabiliT): technical results and polymethylmethacrylate leakages in 25 patients. Skeletal Radiol 2013;42:113-20.

5. Kim JM, Shin DA, Byun DH, Kim HS, Kim S, Kim HI. Effect of Bone Cement Volume and Stiffness on Occurrences of
Adjacent Vertebral Fractures after Vertebroplasty. J Korean Neurosurg Soc 2012;52:435-40.

6. Zhang L, Wang J, Feng X, Tao Y, Yang J, Wang Y, et al. A comparison of high viscosity bone cement and low viscosity bone cement vertebroplasty for severe osteoporotic vertebral compression fractures. Clin Neurol Neurosur 2015;129:10-6.

7. Baoqiu LI. Percutaneous vertebroplasty for acute severe osteoporotic vertebral compression fractures with high viscosity bone cement. Laser J 2013;34:118-9.

8. Guo D, Cai J, Zhang S, Zhang L, Feng X. Treating osteoporotic vertebral compression fractures with intraosseous vacuum phenomena using high-viscosity bone cement via bilateral percutaneous vertebroplasty. Medicine 2017;96:e6549.

9. Nieuwenhuijse MJ, Muijs SP, van Erkel AR, Dijkstra SP. A Clinical Comparative Study on Low vs. Medium Viscosity PMMABone Cement in Percutaneous Vertebroplasty: Viscosity Associated with Cement Leakage. Spine 2010;10:S118-9.

10. Nieuwenhuijse MJ, Van Erkel AR, Dijkstra PS. Cement leakage in percutaneous vertebroplasty for osteoporotic vertebral compression fractures: identification of risk factors. Spine J 2011;11:839-48.

11. Yang DT, Zhang D, Arola DD. Fatigue of the bone/cement interface and loosening of total joint replacements. Int $\mathrm{J}$ Fatigue 2010;32:1639-49.

12. Rotter R, Schmitt L, Gierer P, Schmitz KP, Noriega D, Mittlmeier $\mathrm{T}$, et al. Minimum cement volume required in vertebral body augmentation-A biomechanical study comparing the permanent SpineJack device and balloon kyphoplasty in traumatic fracture. Clin Biomech 2015;30:7205 .

13. Cui W, Liu B, Wang L, Kang W, Chen B. The correlation analysis of balloon volume and bone cement volume in percutaneous kyphoplasty. Chin J Surg 2015;53:289-93.

14. Chen HG, Chen JP, Liang HP, Kong QZ, Chen JH, Zhou Y, et al. Retrospective study on volume of bone cement injection for concurrent of fracture after thoracolumbar vertebraes kyphoplasty. Chin J Orthop Trauma 2012;25:681-83.

15. Kim JM, Shin DA, Byun DH, Kim HS, Kim S, Kim HI, et al. Effect of Bone Cement Volume and Stiffness on Occurrences of Adjacent Vertebral Fractures after Vertebroplasty. J Korean Neurosurg Soc 2012;52:435-40.

This is an open access article distributed under the terms of the Creative Commons Attribution-NonCommercial-ShareAlike 3.0 License, which allows others to remix, tweak, and build upon the work non-commercially, as long as the author is credited and the new creations are licensed under the identical terms

This article was originally published in a special issue, "Recent Trends in Biomedical Research" Indian J Pharm Sci 2020:82(1)spl issue1; XX-XX 